\title{
Towards an Understanding of Augmented Reality Extensions for Existing 3D Data Analysis Tools
}

\author{
Xiyao Wang \\ Université Paris-Saclay, CNRS, \\ Inria, LRI; Orsay, France \\ xiyao.wang23@gmail.com
}

\author{
Mickael Sereno \\ Université Paris-Saclay, CNRS, \\ Inria, LRI; Orsay, France \\ serenomickael@gmail.com
}

\author{
Lonni Besançon \\ Linköping University \\ Norrköping, Sweden \\ lonni.besancon@gmail.com
}

\author{
Mehdi Ammi \\ University of Paris 8 \\ Paris, France \\ ammi@ai.univ-paris8.fr
}

\author{
David Rousseau \\ Université Paris-Saclay, CNRS, \\ IJCLab; Orsay, France \\ rousseau@1al.in2p3.fr
}

\author{
Tobias Isenberg \\ Université Paris-Saclay, CNRS, \\ Inria, LRI; Orsay, France \\ tobias.isenberg@inria.fr
}

\begin{abstract}
We present an observational study with domain experts to understand how augmented reality (AR) extensions to traditional PC-based data analysis tools can help particle physicists to explore and understand 3D data. Our goal is to allow researchers to integrate stereoscopic AR-based visual representations and interaction techniques into their tools, and thus ultimately to increase the adoption of modern immersive analytics techniques in existing data analysis workflows. We use Microsoft's HoloLens as a lightweight and easily maintainable AR headset and replicate existing visualization and interaction capabilities on both the PC and the AR view. We treat the AR headset as a second yet stereoscopic screen, allowing researchers to study their data in a connected multi-view manner. Our results indicate that our collaborating physicists appreciate a hybrid data exploration setup with an interactive AR extension to improve their understanding of particle collision events.
\end{abstract}

\section{Author Keywords}

Immersive analytics; 3D visualization; User interface; Hybrid visualization system

\section{CCS Concepts}

-Human-centered computing $\rightarrow$ Mixed / augmented reality; Visualization application domains;

\section{INTRODUCTION}

Virtual reality (VR) allows us to experience remarkably immersive worlds. These environments can be engaging and promise to facilitate tasks that require a high degree of immersionthe psychological state that users experience when they are surrounded by or in an environment that is continuously streaming stimuli [58]—into their three-dimensional content.

Permission to make digital or hard copies of all or part of this work for personal or classroom use is granted without fee provided that copies are not made or distributed for profit or commercial advantage and that copies bear this notice and the full citation on the first page. Copyrights for components of this work owned by others than ACM must be honored. Abstracting with credit is permitted. To copy otherwise, or republish, to post on servers or to redistribute to lists, requires prior specific permission and/or a fee. Request permissions from permissions@acm.org.

CHI '20, April 25-30, 2020, Honolulu, HI, USA.

Copyright is held by the owner/author(s). Publication rights licensed to ACM ACM ISBN 978-1-4503-6708-0/20/04 _..\$15.00.

https://doi.org/10.1145/3313831.3376657
Since the end of 1960s [49], a number of technical setups (e. g., $[14,17,42,52])$ have been introduced and explored by researchers, with recent developments not only coming (visually) close to the vision of a Holodeck [35] but also making immersive experiences available to the general public. From the start, VR hardware has also been explored for 3D data visualization (e.g., $[15,20,25,26,35,48,51])$ and were proven to be more efficient than traditional setups (e.g., a combination of mouse, keyboard, and 2D screen) in many different aspects.

Compared to fully immersive VR environments, augmented reality (AR) offers new opportunities, in addition to also offering immersive 3D stereoscopic data views. First, AR does not transport users to a fully virtual world, allowing them to interact with real-world objects such as traditional input devices (e.g., mouse). Users are thus not forced to use dedicated input devices (e. g., wand, 3D controller) as in most VR settings, resulting in lower learning costs and a large potential to integrate the new environments with existing tools. The latter is essential because domain experts tend to stick to existing analysis tools and are hesitant to transit to new ones, as has been shown in past work [6] and which we also saw in our field observations. We argue this is one of the main reason that current VR-based immersive environments rarely find their way into practical data analysis workflows used by scientists.

In this work we thus investigated a hybrid setup which extends traditional PC-based exploration tools with AR. This setup allows researchers to benefit from the immersion offered by AR technologies, while still being able to use their traditional analysis tools on classical workstations which possess higher computational power. While some past work (e. g., [39,40,45]) and commercial manufacturers (e.g., $[13,24]$ ) already envisioned or explored extensions of $2 \mathrm{D}$ screens with AR, we focus on the specific design requirements for scientific visualization. This domain differs from past studied ones such as VR/AR-supported video conferences and game-play, especially because of a high demand for precision input. We used Microsoft's HoloLens [38] as a see-through AR head-mounted display (HMD) so that users can seamlessly switch from the $\mathrm{PC}$ view to a stereoscopic data representation, and back. We 
duplicated in both platforms a set of visual data analysis features specific to particle physics to achieve a comparable level of functionality. The features are adjusted with respect to the constraints of their rendering space. We then treat both visual spaces as connected views [57], and let users control them using mouse and keyboard devices to avoid a repetitive switching of input devices. To better understand such AR-supported scientific visualization, we then present an observational study about how scientists want to make use of such a hybrid system, with special focus on particle visualization in high energy physics (HEP). We then discuss the potential usage and future design of such settings with respect to the feedback gathered from our study. Our main contribution is thus not the system design but our study. To our best knowledge, we are the first to examine the practical use of immersive visualization to satisfy real needs of physicists. Our results will guide the design of future hybrid visualization systems needed by physicists and scientists with similar 3D data.

\section{RELATED WORK}

We first review related work in immersive visualization environments, before discussing approaches that facilitate the interaction between different visual environments. We conclude this section with a small survey of visualization in particle physics—our chosen application domain.

\section{Immersive and hybrid visualization environments}

In this work we are interested in appropriate interaction design for $3 \mathrm{D}$ stereoscopic views which provide immersive experience $[30,36,40]$. In the past, the responsive workbench [29], occluded virtual reality glasses [46], and CAVEs [14] have been extensively studied because, compared to traditional 2D screens, they better support visual data immersion. Prior work argued that such environments can foster and facilitate interaction with large datasets (e.g., $[19,37,55])$ as well as improving comprehension (e.g., $[18,25])$. For example, Prabhat et al. [43] concluded that, for 3D biological data understanding, users preferred and performed best using immersive environments, compared to non-immersive ones. Usher et al. [56] found VR environments with effective 3D input makes the task of tracing neural circuits in brain more effective and less frustrating compared to traditional tools. FiberClay [25] visualized massive airplane trajectories combined with the geological map in VR headsets. Its evaluation with experts suggested that it favors the discovery of flying patterns that were not usually noticed, leading the authors to conclude that such immersive systems have benefits for the data sense-making process.

With the benefits offered by visual immersion, researchers also suggested using hybrid visualization environments that combine both 2D screen and immersive environments to benefit from both 3D stereoscopic view and traditional analysis information (e.g., 2D slicing and abstract data). For example, Mandalika et al. [33] combined a traditional desktop with zSpace - a 3D stereoscopic screen-for radiologists. Marai et al. [34] studied collaborative systems combining a CAVE2 displaying public contents-divided into a 2D wall size display part and an immersive stereoscopic display-and one laptop per user to both display private contents and send data to the CAVE. The Studierstube system [50] is a co-located collaborative system which combines one AR headset and one personal hand-held panel. Benko et al. [5] also demonstrated a collaborative case for archaeological data. These past approaches show that the combination of multiple devices can enhance the performance of users and empower them with new types of input by taking the best of each device. Such arguments motivate our own work of extending traditional workstation with immersive environments.

\section{Cross-device interaction}

The communication among multiple devices and the interaction techniques for each device have been studied extensively. Brudy et al. [11], e. g., surveyed papers from the ACM DL up to May 2018 about cross-device computing taxonomies and gave a detailed list of interaction techniques for different input modalities. As our ultimate vision of a seamless integration of the AR extension into the scientific workflow, we are interested in understanding the possibility of using a common interaction technique to control both sides without switching the devices. Using a head-mounted AR device as a workstation extension, Serrano et al. [45] designed Gluey to unify the different devices' interactions for general workflows. In 2015, Microsoft envisioned using their HoloLens to extend the 3D modeling tool Autodesk Maya [24]. They allowed users to control the data in both the desktop and the AR space using the mouse. Millette and McGuffin [39], although they added a smartphone, also kept mouse in a hybrid system for 3D CAD working scenario. In another example, a bank envisioned a scenario where users sit on a desk, pull things from the screen to space, and interact with them using gestures and voice command [13]. While these possibilities have been demonstrated, their benefits and limitations remain unclear as well as how to properly design the interaction, specifically with respect to $3 \mathrm{D}$ data exploration needs. We thus based our prototype on mouse control for both the 2D and 3D views to better understand how that could benefit scientists and how it should be implemented.

\section{Visualization and data exploration in particle physics}

In our application domain of particle physics, visualization is essential for both collision exploration and public education [4]. For example, experts use statistical tools to identify both strange (whose trajectories are hard to explain by current physics laws) and interesting (those who carry a high energy) particle traces, and visualization is needed to understand both. Various tools already support the interactive visualization of particle collisions for different tasks. For example, at CERN, VP1 [27] (Fig. 1) displays experiments happened inside the ATLAS (A Toroidal LHC ApparatuS) detector for searching elusive dark matter particles, AliEve [41] visualizes events of ALICE (A Large Ion Collider Experiment), and iSpy [1] is used for CMS (The Compact Muon Solenoid) detectors. Most of these tools are traditional PC or web-based with several identified defaults: a typical difficulty is that dense events are difficult to visualize due to the overlapping of trajectories after the projection on the 2D screen. VR recently attracted CERN researchers' attention: it is being gradually recognized by physicists that stereoscopic views can help them to understand their data. Yet the few existing tools (e.g., ATLASrift [53] and Belle II [16]) are mostly limited to public education. We, in contrast, investigated the use of AR headsets for data analysis 

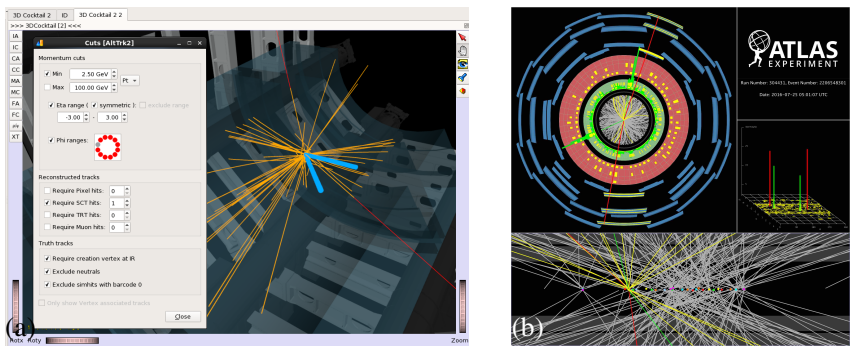

Figure 1. Screen shots of Virtual Point 1 visualization software. Images from the ATLAS Experiment $\odot 2019$ CERN, used with permission.

(a)

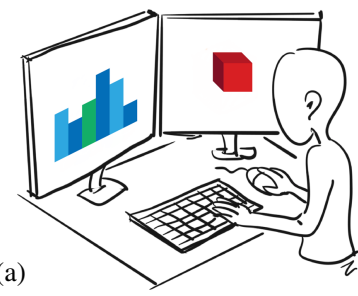

(b)

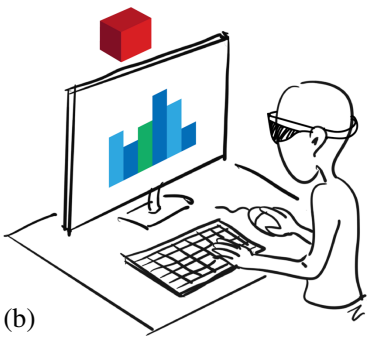

Figure 2. Sketch of our vision of transitioning from (a) a traditional to (b) an AR-augmented data analysis environment. Also see [54].

as they do not occlude users from the real world and thus can augment the 3D views of current analysis tools.

\section{DESIGN CHOICES}

Benefits of visual immersion demonstrated in previous literature and physicists' interests motivated our study. We based our prototype on discussions with our collaborators at CERN (from the Atlas project) to understand their workflow, current tools, and interaction requirements. Through our discussions we learned that their analysis does not only rely on visualization software, they need to switch between data analysis to find and limit the region of interests, and visualization to observe and understand the exact phenomenon. We thus need to consider a system that allows both immersive visualization and traditional data analysis. Moreover, it needs to be easy to setup such a system in an office to be used in the scientists' regular workflows. Based on these considerations, we propose a hybrid setting that combines a PC and a HoloLens (Fig. 2; [54]): We do not aim to replace the existing tools of scientists but rather to propose to use a 3D stereoscopic extension that allows them to better perceive their 3D data, seamlessly combining the benefits of the stereoscopic view with traditional analysis tools. We now explain our choice in detail and the relevance of our study to the domain.

Input. We rely on mouse and keyboard as input devices for both PC and the HoloLens. Physicists' analysis (in contrast to visualization) heavily relies on script writing where mouse and keyboard are essential. These devices are thus important to keep in our hybrid system as we want to integrate the 3D extension into their workflow. In addition, previous work pointed out that experts still prefer traditional input even if new forms of intuitive input exist, for example, studies with fluid dynamics researchers [6] and doctors [33] - similar to the wellknown Legacy Bias in interaction design where "users resort to well-known interaction styles even when more effective and novel techniques are available" [11].
For AR input, even though mid-air gestures are popular means, it has been argued that they could introduce user confusion, error, and fatigue (e. g., [18]). We thus do not envision its use for scientific visualization where high interaction precision is required. In addition, we are interested in unifying interaction design such that users do not need to switch between different input devices as others argued in the past, including for the HoloLens [24]. But such interaction remains a challenge in purely virtual spaces [22]. Our work is thus a step toward better understanding how hybrid virtual environments can enhance scientific analysis and how to improve scientists' workflow with the commonly used input of mouse and keyboard, as well as how such systems should be realized.

Output. We selected an AR HMD because it is light-weight and can easily be used in an office, without occluding the real world as a VR HMD. Users can seamlessly use their traditional analysis tools and benefit from stereoscopic rendering. We thus do not need to recreate all analysis tools as in VR, nor do we need to introduce additional VR-specific input devices. Although AR devices with an additional mouse and keyboard input may fully replace the PC one day, we still explore and study the equivalent PC-AR hybrid setting due to its currently higher fidelity. Moreover, relying on a PC likely will always have merit due to its its high computational power and superior high-resolution screens. We excluded large environments like CAVEs due to their high demand for space and maintenance, which limit a practical integration to regular workflows. We do not use static 3D screens because the virtually unlimited cavas of AR HMDs can provide additional advantages for scientific visualization as such tasks often require multi-view analysis. They are also more flexible to allow users to arrange views to perceive both the 3D space and non-spatial information at the same time. While 3D screens with appropriate interaction techniques can also offer large canvases, we believe that gaze-based view-switching has potentials that we should study. Although we did not investigate collaboration in this work, an AR HMD may also facilitate collaborative data analysis [44] which should be studied in the future.

Study relevance. Although research in AR/VR with multiview settings exist (e.g., [32]), the interaction requirements of 3D data visualization usually differ from those in other use cases, specifically the demand of high-precision work. For 3D selection, e. g., common methods like ray-casting are unsuitable for scientific datasets because they usually do not natively define objects or regions [9]. Another example is the common use of orthographic projection for precise comparison of parallel structures. It is thus important to study such tasks with domain experts to understand the needs and to conduct design guidelines to bring immersive visualization environments into their workflow. Another reason is that, while some have envisioned hybrid PC plus AR interaction as mentioned above, it still remains unclear how domain experts would want to use such a hybrid system, how an AR extension can support data exploration, and how the interaction/UI should be designed to support their needs. To study it, a working prototype is necessary because paper prototypes with flat images remove the immersion provided by AR, thus several features of AR like walking around would be impossible to investigate. 


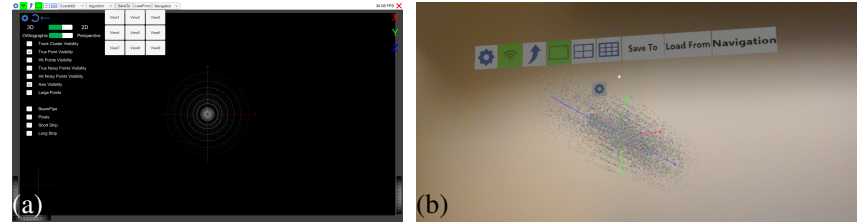

Figure 3. Example of user interface on (a) a PC and (b) the HoloLens. Both of them use a menu bar on the top of the view.
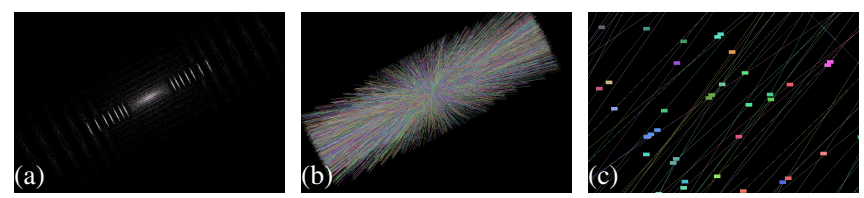

Figure 4. Visualization of (a) hit/true points, (b) constructed/true trajectories, and (c) zoomed trajectories with points in our PC prototype.
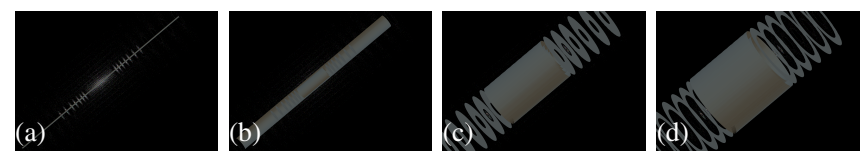

Figure 5. Visualization of (a) beam line as well as (b) pixel, (c) short strip, and (d) long trip detectors with particle hit points in our PC prototype.

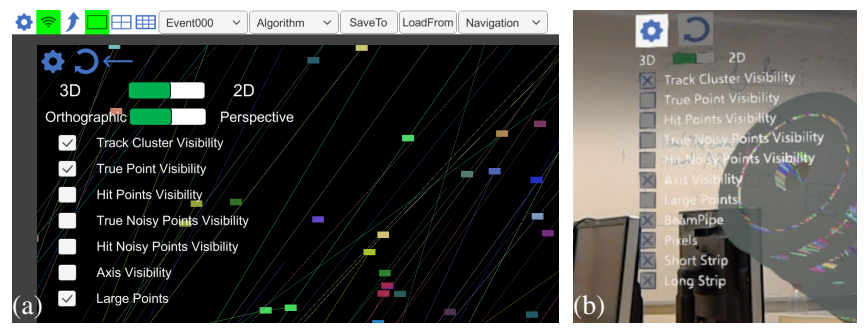

Figure 6. UI Interface: (a) PC and (b) HoloLens.

\section{PROTOTYPICAL IMPLEMENTATION}

We implemented a prototype to serve as an initial tool to understand the potential use case of AR extension with PC data exploration tools-we did not developed it to replace existing software and settings in usability, interaction details, or computing power, all of which are fast changing according to experts' feedback, yet are not the key points we discuss.

Our prototype consists of two parts: one on the PC and one on the HoloLens (Fig. 3), both inspired by our collaborating particle physicists' regular work environments. We envision the metaphor of using a two-screen environment, in which the content of each screen can be defined individually and the mouse can travel from one screen to the other. We then replace one of these screens by the AR environment (Fig. 2). The users can remain seated and continue to work with their traditional tools as usual on their PC or laptop, but can also transition to the AR environment when needed or go back to the $\mathrm{PC}$ at any time. The communication is based on WiFi using the UDP protocol and is bi-directionnal, i. e., motions happening in the AR environment are also transmitted to the PC. We created all implementations in Unity with C\# and its framework . NET. We transmit data via UDP due to its simple implementation and small processing overhead. In a controlled network, data usually arrives in order and without loss.

We then created comparable functionality on both the PC and the AR platform, including the user interface and the interaction logic, i. e., all tasks described in this section can be performed both on PC and on HoloLens. Our general idea with this prototype is that both views share the same dataset but can be configured differently (views, settings, manipulations, etc.) - just like multi-view environments on traditional PC settings. Users can pull the current configuration from one side to another using UI actions. They can also switch the real-time synchronization between both device on and off, to understand how users prefer to use such a function.

Data. We use simulated proton collision events from the MLTrack Challenge [2]. A single event contains information about (1) the true hit points (collision with detector hardware, including position and momentum data), computed through physics laws, (2) the simulated measured hit points (with simulated measuring error), (3) information on the particle' trajectory, to which we refer as a track (Fig. 4). One event contains about $10 \mathrm{~K}$ tracks with $100 \mathrm{~K}$ points. Basing our visualization only on simulated measurements, we connect the points' positions to get the particles' measured trajectories. To reduce the rendering cost, we simply connect the points with straight lines which is also done by the physicists, without introducing ambiguity for understanding the true trajectories.

Mouse transitions. Mouse and keyboard input are captured first on the PC, and then transmitted to the HoloLens. On the $\mathrm{PC}$ we use the mouse as usual. The mouse switches between the 2D screen and the AR space by pressing the Tab key. We did not use implicit transitions when the mouse crosses the screens' borders as done with two 2D screens to avoid unintended switches between the two platforms. Indeed, the borders' area of their traditional tools usually contains UI elements to perform manipulations or to change system setting (Fig. 1(a)) causing users to frequently manipulate this area. We did not use gaze focus to control the cursor either like others [45] because we leave users the possibility to see both the $3 \mathrm{D}$ and the $2 \mathrm{D}$ views at the same time, instead of forcing them use only one. In AR space, the mouse can move-in addition to its 2D motion-along the depth axis with the scroll wheel, while the Shift key is pressed. We we decided to not reinitialize the mouse's depth position after releasing the key (i.e., the mouse will not be back to the default position "in front" of the 3D box). Yet even though the UI widgets in AR are fixed on the box' front, users can still click on them while the mouse is behind them based on ray-casting (without drawing the ray). We added a visual feedback (color highlighting) while the mouse "hovers over" the button.

In the remainder of this section, we list and discuss how we solved the requirements pointed by the experts. We also report our insights from analyzing their current tools.

Following the track. Physicists want to follow a single track while exploring, thus each of them need to have a unique color. Their current tool does not provide a standard color map for all tracks, we thus created one to make sure that they are not too bright to hurt eyes in either space.

Abstraction for data and detector. It is essential for our collaborators to visualize both the detector structure and the collision data. The Atlas detector comprises three main parts: inner detector, calorimeter, and spectrometer. In this study, we 

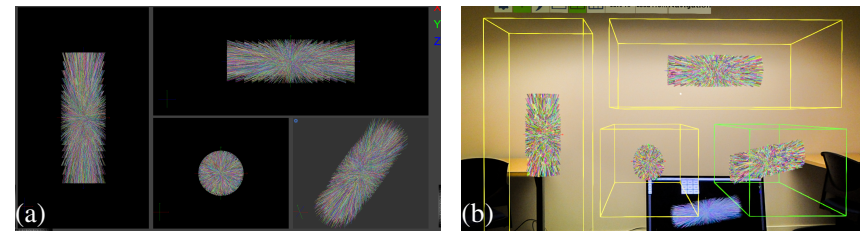

Figure 7. Visualization of $2 \times 2$ views: (a) PC and (b) HoloLens.
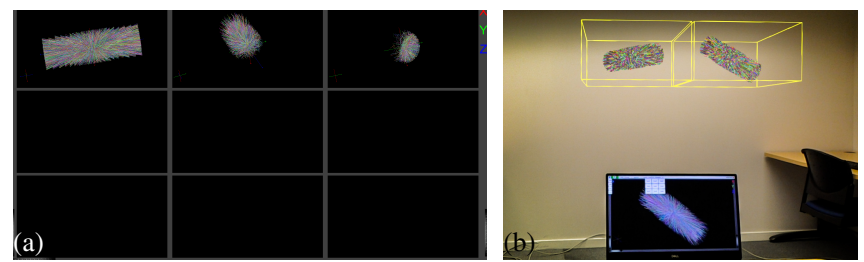

Figure 8. Visualization of $3 \times 3$ views: (a) $\mathrm{PC}$ and (b) HoloLens. Only saved views are displayed.

use a simplified model with only the meshes of the beam line (where collision happens) and inner detector (which includes pixel detectors, short strip detector, and long strip detector, see Fig. 5). Switching on and off different detectors is triggered using UI widgets (Fig. 6), similar to their existing tools.

Visualization of different perspectives. Domain experts need to compare different views of the same data. As shown in Fig. 1, the current tool uses multiple windows or several views on the same screen. We chose the latter as AR has a larger canvas that supports the simultaneous rendering of multiple views. We implemented $2 \times 2$ views (Fig. 7) including the front, top, left, and perspective views as commonly found in many $3 \mathrm{D}$ tools. Those views have different transformation (position, rotation, and scaling), but share the same dataset: manipulations such as filtering are applied to all of them. Those views are not restrained to pre-defined settings, each of the them is configurable. Users first click on a specific view, and then can change its setting (in Fig. 7, e. g., the right bottom view's is highlighted to indicate that users interact with just this view).

Save and jump to specific views. Domain experts also need to be able to save specific states (including transformation, filtering, and abstraction level) of the dataset they are exploring and may jump back to a former state later. We thus implemented a $3 \times 3$ dump board (Fig. 8) that carries the saved states. The dump board is always synchronized between the PC and the HoloLens, thus users can perform interaction on any view and freely switch to the desired setting on the other.

3D navigation. 3D navigation allows physicists to explore and understand the spatial aspects of the dataset at hand. Their current tool includes interaction with 5 degrees of freedom (DOF): $x$ - and $y$-rotations, $x$ - and $y$-translations, and uniform scaling. However, they are insufficient in an AR setting where users need to translate the data along the $z$-axis to specify its position in space. We thus defined a 7 DOF navigation mapping using mouse and keyboard, derived from one of their used tools and previous work [7] as follows: the left button triggers a rotation around the $x$ - and $y$-axes, the right button triggers a translation along the $x$ - and $y$-axes, the scroll wheel translates along or rotates around the $z$-axis (a single click on
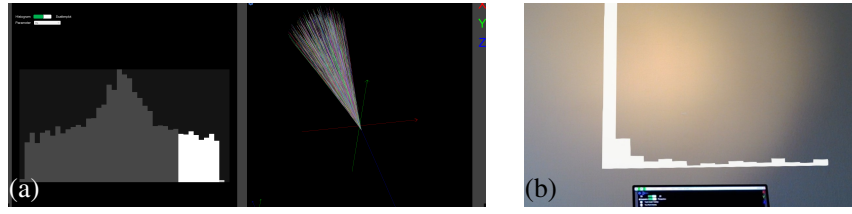

Figure 9. Histogram filtering: (a) PC (by $\eta$ ) and (b) HoloLens (by $p_{t}$ ).
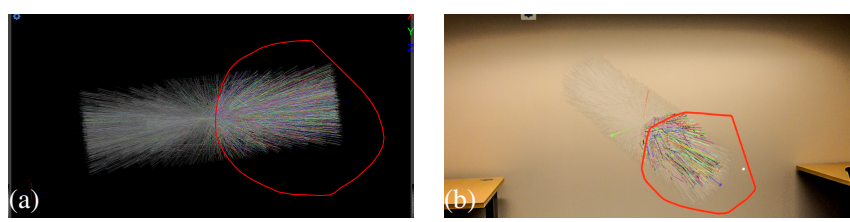

Figure 10. Spatial selection: (a) the PC and (b) the HoloLens.

the wheel switches from one to the other), and scrolling while $\mathrm{Ctrl}$ is pressed triggers zooming.

Selection by parameters. Each particle has many parameters. Experts usually plot histograms and find a region of interest. They then use their tools (VP1 or special Python packages) and focus directly on the special particles by selecting them based on the target parameter values. Based on the previous habit of using histogram, we make them interactive. Users can highlight and filter particles through clicking/sliding on the histograms (e.g., left side of Fig. 9(a)). Explicit filtering is triggered by direct clicking/sliding, while track highlighting is triggered with Ctrl button. In our prototype, we support histograms of following properties: azimuthal angle (phi, $\phi)$ in cylindrical coordinates, pseudorapidity (eta, $\eta$ ) related to the the dip angle in cylindrical coordinates, transverse momentum $\left(p_{t}\right)$, the momentum of the generated particle projected onto the transverse plane, the radius of the production point of the particle $\left(r_{0}\right)$, and the distance of closest approach to the $z$-axis of the trajectory of the particle when extrapolated $\left(d_{0}\right)$.

Spatial selection. Domain experts sometimes need to select tracks based on their positions in their visualization software. We implemented a lasso tool (Fig. 10) which is often found in 3D exploration tools (e. g., [9,59]). Users can apply Boolean operations to intersect, unite, or delete the selected tracks with the visualized ones. Using screen widgets, users can also specify if they want to select particles with all trajectories inside the lasso or with at least one part inside it, thus keeping the complete trajectories of the particles. Selected regions are first highlighted, then filtered after confirmation (Fig. 3).

\section{OBSERVATIONAL STUDY}

To better understand the implications of combining a traditional workstation setting with an AR view and how to develop interaction mechanism for such hybrid environments, we conducted a preregistered (https://osf.io/7qegs/) observational study with seven experts in the domain of particle physics. While this number of participants may appear low, it is not an unusually low number when conducting observational studies to understand the needs of domain experts [6, 8, 28,30,31,47]. We were interested in their general opinion on such a system and their feedback on how the interaction should be designed to better answer the tasks that they have in their domain. We used an observational strategy that has been used 


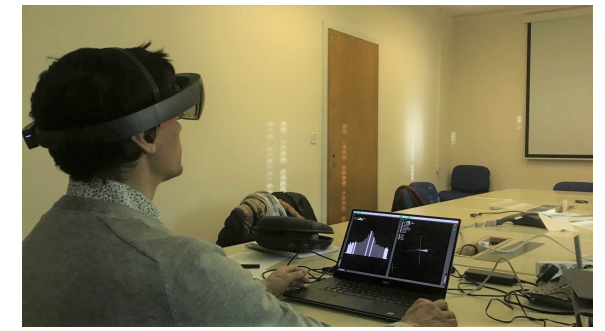

Figure 11. Experimental environment.

by several other researchers and research work before when designing for and reflecting on specific domain experts needs (e. g., [6, 21, 25, 28, 30, 31,47]). We specifically decided not to focus on usability studies and time or error measures for several reasons. First, our goal is to understand how can we extend the current data analysis tools with an AR extension instead of presenting new technique/system, we do not aim at proving a hybrid environment that works "better" than a traditional PC-based 3D analysis tools. Also, we do not want to miss meaningful critique and ideas which could be prevented by using quantitative studies as pointed out by Carpendale [12].

\section{Participants}

We recruited 7 CERN researchers as unpaid participants, all working on HEP and denoted as P1-P7 (6 males, 1 female; ages 26-52 years). They had 2-30 years of post-Master's research experience (mean: 12.4, median: 12, and SD: 9.1). All were used to interact with 3D datasets in their work using typical mouse+keyboard interaction (one reported 1-2 times a week, while all others reported several times a day or that it was basically their daily work). Six of them had knowledge about VR glasses, three had limited experience with immersive environments (only VR glasses), and none of them had experience working with Microsoft's HoloLens.

\section{Apparatus and Setup}

Our prototype used the first version of Microsoft's HoloLens (development edition) and a Dell XPS 9570 laptop $(3840 \times 216015$ ' screen) running Windows 10 with its integrated keyboard and a Bluetooth mouse. We connected the laptop to a local TP-Link AC750 router via Ethernet, to which the HoloLens connected via WiFi. We ran the study in a meeting room at CERN in Switzerland. We placed the laptop on one side of a big meeting table and let the Hologram initially focus on the center of the table, at around 2.5 meters away from the participant and vertically a little higher than the PC screen (near the other edge of the table). As shown in Fig. 11, the room had whitish wall and was lighted as their normal office (the windows on the other side of the wall were closed by blinds). Nobody walked into or out of this room during the experiment. Participants sat on a fixed-leg chair.

\section{Study design and procedure}

The same dataset was used by all participants in our observational study. Each participant performed the experiment individually, in the mentioned room, alone with the observer. Participants were video-recorded (participants are not always visible but audio is fully recorded) for analysis. We started by explaining to participants the purpose of this study. We then asked participants to read through and sign, if they agreed, a consent form, a media-release form, and a questionnaire collecting their demographic information and past experience with 3D data exploration, 3D interaction, and immersive environments. We then began our three-part experiment:

Explanations and tutorials. We first re-stated our purpose with this study and emphasized that the goal was not to show a more impressive system with better usability or functionality compared to the traditional one. We then introduced them with the apparatus. We told them that we would first introduce the user interface and explain the interaction. We also explained that the task was to use all possible interaction techniques to explore the data, before a semi-guided interview to understand how they used our system. After they had put on the HoloLens and adjusted it to a comfortable state, we began the introduction. We did not stream their view to avoid the drop of performance, we asked them to confirm their understanding after each presentation and encouraged them to ask questions themselves. In our design scenario, users sit down and work on their data as in their office. However, walking around is a feature of AR HMDs that is under-explored in visualization. We thus told participants to feel free to get up and walk around.

Free exploration and thinking aloud protocol. Once we finished the explanations and the participant was ready, we left full control to the participant. We asked them to interact with the system using all implemented functions and freely explore the described simulated collision dataset. Participants were allowed to ask for help or pose questions while exploring and were encouraged to think aloud, i. e., to express directly what they observed and thought. The experimenter observed the whole experiment next to the participant, took notes, and guided the participant if they felt that the participant forgot something at the end (e.g., if the participant used only some of the features, we encouraged the participant, without forcing them, to try others as well). There was no set time limit.

Questionnaires and semi-guided interviews. When a participant reported that they had finished exploring the dataset, we asked them to take off the HoloLens and take a short break. We then conduced a semi-guided interview asking their general feedback about the AR extension and the interaction with mouse and keyboard, as well as any potential improvements they envisioned. We finally asked participants to answer 5- or 7-point Likert questions to quantify some of their ratings. To fairly discuss the potential usage, we highlighted again, before the interview, that our system was a prototype whose purpose was to understand how to use an AR extension, instead of presenting a new system. We also explained that many current hardware limitations would be improved with the release of new AR devices, thus highlighting that they should focus more on the interaction and visualization aspects.

\section{RESULTS}

Participants took 30-60 min to finish the exploration, and 1.5-2 hours for the whole study. We report the collected quantitative data and qualitative insights next. Because they complement each other, we report these two types of results 


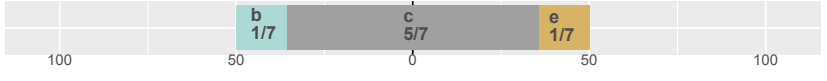

Figure 12. "For practical data analysis in my future daily work, I would prefer:" (a) only a PC interface, (b) in-between, (c) half-half hybrid interface, (d) in-between, or (e) only an AR/VR interface.

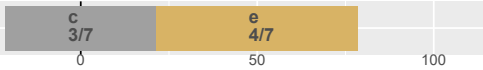

Figure 13. "In an interface that uses AR/VR stereoscopic elements, I would prefer:" (a) a VR interface that only shows virtual elements and where the real world is completely invisible, (b) in-between, (c) I have no preference, (d) in-between, or (e) an AR interface in which the virtual elements are shown in addition to the real world.

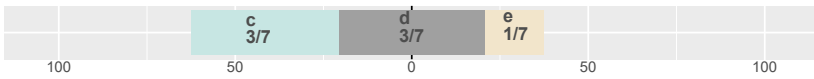

Figure 14. "In general for 3D data analysis (not necessarily your own work):" (a) the PC is the best platform, (b) the HoloLens is an interesting yet not particularly useful addition the $\mathrm{PC}$, (c) the HoloLens is a nice addition to the PC that is sometimes useful, (d) a balanced combination of $\mathrm{PC}$ and HoloLens is best, (e) the $\mathrm{PC}$ is a nice addition to the HoloLens that is sometimes useful, (f) the $\mathrm{PC}$ is an interesting yet not particularly useful addition the HoloLens, or (g) the HoloLens is the best platform. P1 did not vote while P4 voted for 2 options.

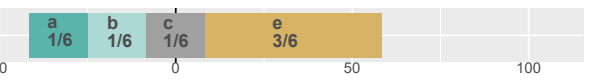

Figure 15. "For my typical data analysis at CERN/of particle collision data, the forced perspective view on the HoloLens/AR view is" (a) not useful at all, (b) in-between, (c) somewhat usable yet has some perspective projection issues, (d) in-between, or (e)completely equivalent to orthographic views on the PC. P4 did not vote.

together, organized into several categories. ${ }^{1}$ Many of the questions are exploratory (i. e., we ask about their ideas without letting them try other environments) as several have experienced VR before and our goal is to learn about their general opinions of the most suitable immersion experience for such analysis tasks instead of precisely comparing the usability.

Interface. We asked participants about their general impression of such system and if they would prefer an interface with only the PC, an interface using only the HoloLens or another VR headset, or a combined one as we showed. P1 reported a high preference of using only an immersive interface and P2 suggested a hybrid system but with more focus on the PC side, while all other participants preferred a half $\mathrm{PC}$ and half AR/VR hybrid system for their future tools as shown in Fig. 12 and Fig. 13. We report specific comments below.

Pl did not see the point of keeping the background environment visible. Then, with proper input, a pure VR environments would be fully capable for exploring such datasets. Background information may also be source of disturbance, imaging people walking around. However, the use of AR facilitated the combination with the laptop which is highly advantageous, and there is no sickness feeling as in VR.

$P 2$ agreed that the PC can be used to manage expensive computation, while visualizing the results in AR space.

P4 thought that the HoloLens is less disturbing than VR headsets by not occluding the real world. The PC worked better

\footnotetext{
${ }^{1}$ We based the plot of Likert-scale results on http://rcompanion. org/handbook/E_03.html
}

for precise interaction and abstract data visualization, while AR offers better depth perception for 3D visualizations.

P5 preferred a hybrid system, but if a future HoloLens becomes powerful enough he can imagine the scenario of using only the HoloLens for both 3D and 2D visualization, potentially with a virtual keyboard.

P6 preferred a hybrid system because laptop could be kept for practical data analysis tasks.

$P 7$ was more interested in stereoscopic visualization than regular $2 \mathrm{D}$ screen projections.

Besides, P7 noted that scenarios may exist in which the PC and the HoloLens would better be used separately depending on the tasks. She explained that switching the focus both for visualization and interaction between the $2 \mathrm{D}$ and $3 \mathrm{D}$ space can be annoying, while all other participants did not share the same feeling. Nonetheless, P3 mentioned that switching was unconfortable at beginning, since he is not used to look up and down because his office screens are aligned horizontally.

We then asked about the roles of each platform, see the results in Fig. 14. P1 did not give any preference because he would personally prefer a VR environment instead of an AR, PC, or hybrid system. P3 and P7 thought that the HoloLens is a nice addition to the PC that is sometimes useful, but the major tasks would still be performed on PC. P3's justification is that all the same things can be displayed on a $2 \mathrm{D}$ monitor and that experts are quite trained to understand perspective there. P5 and P6 prefer a balanced combination of PC and HoloLens. P4 reported two preferences (c) and (d). He thought that, in principle, a balanced combination is perfect for data interaction, but that it would depend on future performance of the hardware: Today's limitations of the HoloLens mean that it can only be seen as an addition to the PC to use occasionally.

Perception and data understanding. We asked about the difference of perceiving data between the HoloLens and the 2D screen. P7 reported that the AR space is similar to an additional 2D screen while other thought that AR provides more than an additional screen, especially emphasizing its added depth perception. We report specific comments below.

P3 appreciated the HoloLens's low resolution in some cases as it makes certain data elements bigger, such as particle hits and curve trajectories which are often tiny. In addition, AR systems would allow a better understanding of detector structures and their spatial arrangement with the particle trajectories, which will largely help at understanding events.

P6 expressed that the direct interaction with the data to see how tracks go from one vertex to another is impossible or, at least, hard to achieve on the PC.

$P 7$ could not perceive the data close to her due to the narrow field of view of the HoloLens. She then needed to move the data further away, which limits the 3D immersion.

Data visualized in AR space is forced to be shown in a perspective view (to maintain stereoscopy), but 3D visualization software often relies on both orthographic and perspective views. We tried to understand how experts feel about this forced yet physically correct perspective projection and the 

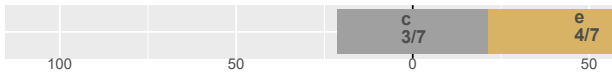
e
$4 / 7$
50

Figure 16. "In an improved interface, I would prefer:" (a) the PC and HoloLens views always in sync, showing the same exact views and selections, (b) in-between, (c) the PC and the HoloLens showing different 3D/2D views yet with the same dataset and the same set of filtered/highlighted tracts, (d) in-between, or (e) the PC and HoloLens views completely separated, showing different views, different selections, maybe even different data, like two independent applications.

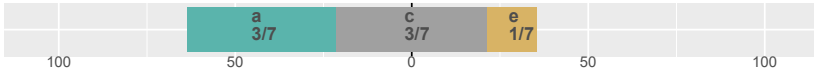

Figure 17. "For interacting with the HoloLens/AR view (e. g., selections), I would like to use" (a) completely gesture/hand based input, (b) inbetween, (c) input that combines hand and device actions such as a pen to point directly at a track, standing next to it, (d) in-between, or (e) fully hardware-based input where the device is separated from the 3D HoloLens view and that only uses a virtual pointer.

mismatch to a potentially shown orthographic projection on the PC. Fig. 15 summarizes their general opinions. P1-P6 reported that perspective projection on HoloLens is just natural, they did not see why orthographic projection is useful in AR space for experts. We report other comments below.

$P 3, P 4$ said that as domain experts who understood the data well, the link between the orthographic projection on PC and the forced perspective projection in AR is easy to make.

P4 did not vote because he thought the perspective in AR is more useful than orthographic view, but he would keep the orthographic views on the PC side.

P5 understands better events with visualization, yet he typically does not need to do measurements in visualization software, thus keeps perspective views which are enough.

Synchronization. Understanding the synchronization between the $\mathrm{AR}$ and $\mathrm{PC}$ views gives insights on how to design and use such a system. P1-P4 would prefer to have the two spaces totally separated and self-configurable, like two different applications. P5-P7, instead, would prefer keep the two different views yet which both reflect the same state and dataset. We present other individual preferences below.

P1 prefers to have two different views/interfaces. While not synchronized, it looks weird if the two views have the same interface but do not show the same content.

$P 3$ has multiple preferences, depending on the application. If he works alone, he would prefer both sides to be configured separately, while he would keep them synchronized for public presentation and collaboration.

$P 4, P 5$ prefer to have switchable configuration by users. In one case, one can work on the PC and see the changes directly on the HoloLens; in another situation, they would keep the one state on one side and to then be able to easily compare the different states.

$P 6, P 7$ were confused when the two views had the same interface but were not synchronized.

Input. All participants agreed that the AR space should not be used only as a static screen, but be interactive using better interaction techniques as Fig. 17 shows. In our study, however, we saw that certain implementation limitations can play a big role in the perception of user input devices. Specifically, our participants did not appreciate the current state of the mouse and keyboard input because, in particular, the mouse movement was sampled and transmitted to the HoloLens at the frame rate of the program. This caused the mouse movement to not appear smooth and instantaneous on the HoloLens, compared to the PC. Besides these solvable technical issues,

$P 1$ is familiar with the mouse which is good for precise interaction, but seems not interesting to be imported to AR.

P1, P4, P6 want a real 3D mouse for AR.

$P 2$ may be interested in spatial 3D trackers and joysticks.

$P 3$ is more used to touchpad or touch screen input.

$P 5$ is more interested in $3 \mathrm{D}$ mice, while moving $2 \mathrm{D}$ ones in $3 \mathrm{D}$ is useful as we can easily click on something.

Both P3 and P7 thought that 2D gestures on touch pad or screen could facilitate the interaction from their previous experience, especially for zooming. We also observed that all participants used a lot of zooming while exploring the dataset. Although we saw a preference for investigating the use of a 3D mouse, P6 envisioned other problems for such input because a 3D mouse can be hidden behind or inside the data. Another difference compared to $\mathrm{PC}$ is that an $\mathrm{AR}$ view has almost unlimited space, so that the cursor can get lost due to a fast interaction (but also because of the narrow field of view).

All participants were willing to use hand gesture input in AR:

$P 2$ thinks that they are less precise but natural.

$P 4$ felt the urge to touch the hologram in space.

$P 5$ would not use them all the time as they can be fatiguing. However, he wants to enable gestures for certain tasks, complementary to a hand-held device.

P6 is interested to navigate and select with hands and fingers, but only if they are accurate enough.

$P 7$ would use them on the HMD and use a mouse for the PC.

No participant wanted to use voice commands due to, e. g., the problems in office settings and possible accent issues. Also, no one revealed specific comments on the gaze control.

Walking around or remaining seated. Getting up and walking around the data using the HoloLens or remaining seated and using a rotation/translation interactions are often discussed with AR setup (e. g., [18]), we present results in Fig. 18. During the experiment, participants mostly sat on the chair, but had a few attempts to get up. P3-P6 thought that walking around or into the data could be quite helpful for data understanding. We report other individual comments below.

P1 sees no point of walking around, which is especially limited in an office. For him, regular interactions are sufficient.

$P 2$ is wiling to walk, but it may be useless unless other input is supported since mouse\&keyboard are not carryable.

P4 prefers moving his head to walking or mouse-based data manipulation. He sees the gaze-based exploration as a main difference to the PC, yet it is less practical in an office.

P6 sees it as the HoloLens' main advantage. However, analysis will be interrupted due to disconnections with the PC. 


\section{\begin{tabular}{|lll}
$\mathbf{b}$ & $\mathbf{c}$ & $\mathbf{d}$ \\
$1 / 7$ & $1 / 7$ & $1 / 7$
\end{tabular}}

100

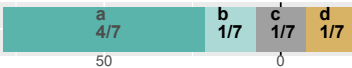

Figure 18. "Seeing the AR stereoscopic data from different sides and perspectives," (a) I prefer getting up and walking around the data view, looking at the data from different locations, (b) in-between (c) I like both, (d) in-between, or (e) I prefer remaining seated, using rotation/translation to see the data from different vantage points.

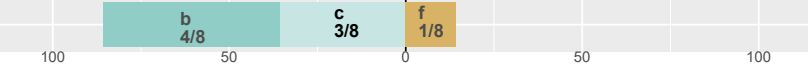

Figure 19. "In the future I could envision to use a hybrid AR interface" (a) 8h a day (b) a few times a day (c) a few times a week (d) 1-2x per month (e) 1-2x per year (f) not at all. P4 voted for 2 options.

P7 was disappointed due to the limited field of view.

Application and collaboration. We asked participants about their envisioned application and collaboration scenarios, supposing there are no hardware barriers. P1, P2, and P4-P6 stated that a hybrid system could be interesting for collaborative meetings. Further comments included that

$P 1$ finds it interesting to view the data as well as messages from others. Unlimited space could help collaboration.

$P 2$ envisioned the system to be useful to present to others.

$P 3$ similarly, specified that the system would be useful for general public presentations but not for experts.

P4 thought that, during collaboration, only one person should interact with the data at a time, while others only observe.

P6 would allow a larger but finite number of users to interact.

Realistic usage in daily work in the future. We summarize our participants' envisioned future amount of use of a hybrid setting in Fig. 19. P7 sees no realistic usage in her daily work since visualization is not her primary task while others disagree. However, to make such hybrid system realistic, P1, P3, and P6 would expect other specific functions. Even though they all work in high-energy physics, their work requirements differ significantly. P4 voted for two options because he thought the realistic usage would depend on whether the task requires more analysis or visualization. He detailed, e. g., that it should have a way to import, export, or communicate the model and settings with other software.

Comments of each platform. We report some other comments regarding the HoloLens and the PC.

Pl appreciated the large AR canvas which facilitates working on several things simultaneously. However, the PC has easier accessibility and usability: everyone knows how to use the PC mouse and interact with standardized UI elements.

$P 2$ sees that the additional spatial dimension shown by the HoloLens allows people to see the data in a more intuitive way (e. g., needing less rotation that on the PC). However, people are well trained to use and understand data on PCs.

P3 thinks that the HoloLens improves 3D perception because we can "walk into things" and arrange elements in space.

P4 commented that the AR visual immersion improves data understanding, and that the $\mathrm{PC}$ is good for precise control and the display of abstract information.
P6 believes that the PC is good for quick input such as typing, its high resolution allows us to display more details. Its familiarity is also an advantage.

P7 appreciated the HoloLens' better depth perception, but thinks that interaction is easier on PC.

\section{DISCUSSION AND LIMITATIONS}

While our participants' responses to our set of questions as reported in the previous section already provide a lot of inspiration for the future development of AR-supported data exploration environments, we now discuss them in the context of our overall vision of a hybrid system.

Lessons learned. We observed that due to our design of the AR-part of the interface to match that of known tools, all our participants quickly understood how to work with both parts of our hybrid setting — no participant expressed a fundamental uneasiness about the new design. While this is not necessarily surprising, it suggests that such a design may lead to higher adoption rates than VR-only setups. Five of our seven participants stated that they would use a hybrid system instead of pure AR/VR or pure desktop systems. However, the placement of the stereoscopic views needs more consideration to avoid disturbing existing (horizontally aligned) screen layouts.

The AR view was clearly seen as complementing the $\mathrm{PC}-$ most of our participants, like us, do not expect it to fully replace traditional workstations. Some participants expressed that they would carry out certain types of analysis (script writing, abstract data visualization) on the PC, while they would prefer 3D inspection on the AR view. For the synchronization between PC and AR the opinions were diverse, people suggested scenarios where constant synchronization could be useful as well as other situations were the displays should be akin to separate applications. The possibility of getting up and "walking around or into the data" was evidently quite novel to our participants, but we suspect that people hesitated due to the novelty of this form of data inspection and the environment of an office not inviting such actions. When we developed the system, however, we had observed that one of our collaborating domain experts did get up on his own accord to look at the data "more closely" in an intuitive way. So we believe that this is an exciting possibility for analyzing 3D data.

The main advantage of AR to our participants seemed to be its virtually "unlimited space"- not only for 3D content but also for the visual comparison of other views. Future work should thus investigate how to best make use of this space. Following our initial vision, the AR view could potentially extend existing tools by providing both screen space and stereoscopic views of the 3D data, yet in a fundamentally different way than another 2D or auto-stereoscopic screen on the desk.

Nonetheless, the AR extension is not seen as simply a static 3D stereo view, instead people feel a strong desire to interact with it. Our simple replication of the 2D mouse in AR space did not feel comfortable to people - partially due to the mentioned technical limitations. Yet even if these problems were resolved, it seems that a dedicated input device such as a 3D or space mouse may be more useful. After all, we also change from keyboard to mouse and back during interactions with regular 
workstations so another dedicated input device may not feel as disturbing as one may expect. This device would need to provide similarly precise input like a regular mouse in $2 \mathrm{D}$ to support the precise interactions needed for data analysis. We also would need to understand better how to use a "HoloLens cursor"-it should be inspired by the 2D counter-part yet may need special functions to avoid, for instance, it getting lost in the mentioned large AR interaction space and to always be visible, even in dense data situations.

While our participants mentioned several other possible forms of input such as hand gestures or joysticks, we are hesitant about such designs without empiric evidence that these would provide as much flexibility, control, and precision as a mouse. In particular gestures in empty space-even if envisioned by our participants to be intuitive and "natural"-can quickly become tiring due to the gorilla arm syndrome [23]. One interesting and promising idea, however, is the use of gestures on a potentially existing (laptop) touchpad as they are currently used to augment the interaction in traditional interfaces. Certain well-defined multi-finger gestures for 3D navigation (not only but including two-finger pinch-to-zoom) could be an excellent form of input for the AR space.

Much to our surprise we found that the lack of orthographic projection in AR did not bother any of our participants, despite the prevalence of orthographic views in traditional 3D data analysis tools like the ones used in particle physics. In the future we thus would be interested in studying whether AR views with correct perspective are similarly precise and efficient as orthographic projections (within the domain of particle physics and elsewhere) for solving tasks in 3D space because this is a fundamental prerequisite for an effective 3D data analysis. This apparently "correct perception of 3D shapes" may also shed light on the limitations and benefits of 3D representations of abstract (i. e., non-spatial) datasets [10].

Finally, our participants' suggestions to use an AR-augmented environment for collaboration appears to be straight-forward, yet also raises numerous interesting research questions. In addition to known challenges of collaborative work, we would be interested in how people would actually physically immerse themselves into 3D data representations by walking around in the views, and to what degree this could support data analysis tasks in our application domain of particle physics.

Generalization. Our study was based on a PC and a HoloLens using particle physics datasets. However, we believe that our findings can be generalized. Any setup that combines a PC with some sort of stereoscopic 3D display can benefit from our discussion on how to add immersion to existing workflow, but with some limitations regarding the walk-around feature and the management of multiple users in collaborative scenarios. As we stated in our design choices, our results also hold in a possible pure-AR environment which makes use of physical mouse and keyboard. The question remains whether our observations for our specific application domain generalize to other domains, specifically since the daily tasks of our seven participating experts already differed significantly. Yet our participants and anybody dealing with some form of 3D data have to carry out at least the same fundamental 3D manipulations techniques and use similar visualization tools. The tasks for analyzing spatial data are comparable to other applications such as air traffic control [25], and even 3D visualizations of non-spatial data [3] require similar forms of interaction. We thus believe that our observations can generalize to or at least inform the interface design in such related domains. Practitioners can thus build on our findings to build hybrid systems more specifically adjusted to specific visualization and data exploration needs.

Limitations. In addition to the known limitations of the hardware of the HoloLens 1.0, our prototypical implementation had limited functionality and the communication between the input devices and the AR view exhibited the discussed lag. However, the hardware and software setup only served as a basis to investigate the future design of a more complete solution, futures iteration will focuse on a more specific set of tasks within our application domain. We expect that improved AR hardware will remove some of the known technical shortcomings (e. g., limited field of view, low resolution), yet its handling will be similar to the present version. Naturally, the specific experiment design and, in particular, the use of think-aloud in the presence of an observer has the potential to bias participants. Yet our study design has established way to conduct observational experiments to extract people's feelings and ways of thinking about technology. We believe that with this approach we obtained much richer input for creating novel hybrid interaction designs than we would have with any controlled speed-and-error experiment. Finally, while we emphasized during the experiment that we did not want the physicist to focus on the technical achievements that the Hololens represent, it is still possible that this novelty effect might have biased their subjective answers. To address this issue we will continue to collaborate with a number of experts in particle physics and work toward a more refined design of a hybrid system that is more usable for actual data analysis and through this continued collaboration we expect to be able to conduct more controlled experiments after our collaborators have gotten used to the new devices.

\section{CONCLUSION}

With this paper and the results from our observational study, we provide insights on how to bridge the chasm between the potential benefit of immersive environments and the lack of adoption in the domain sciences. Results suggest that, first, scientists strongly favor hybrid AR setups where the AR complements the PC. Second, content in the two environments should not constantly be linked. Third, walking through the data is fundamentally more intuitive than view manipulations. And the view-based access to lots of virtual screen space is one of AR's main advantages. We thus open up a completely new form of immersive system design for visualization: we no longer need to decide between immersion and existing tools, but we can use the best of both worlds. Such insights are not limited to particle physics, practitioners working with similar 3D dataset can benefit from our results to extend their data analysis tools with immersive views.

\section{Acknowledgments}

We thank the physicists from CERN for their valuable time and feedback, Pierre Dragicevic and Steve Haroz for their feedback on the paper, and Yumin Hong for the sketch. 


\section{REFERENCES}

[1] George Alverson, Giulio Eulisse, Thomas McCauley, and Lucas Taylor. 2012. iSpy: A powerful and lightweight event display. Journal of Physics: Conference Series 396, 2, Article 022002 (Dec. 2012), 6 pages. DOI :

https://doi .org/10.1088/1742-6596/396/2/022002

[2] Sabrina Amrouche, Laurent Basara, Paolo Calafiura, Victor Estrade, Steven Farrell, Diogo R Ferreira, Liam Finnie, Nicole Finnie, Cécile Germain, Vladimir Vava Gligorov, and others. 2019. The Tracking Machine Learning challenge: Accuracy phase. arXiv preprint 1904.06778. https://arxiv.org/abs/1904.06778

[3] Benjamin Bach, Ronell Sicat, Johanna Beyer, Maxime Cordeil, and Hanspeter Pfister. 2018. The hologram in my hand: How effective is interactive exploration of 3D visualizations in immersive tangible augmented reality? IEEE Transactions on Visualization and Computer Graphics 24, 1 (Jan. 2018), 457-467. DOI: https://doi.org/10.1109/TVCG.2017.2745941

[4] Matthew Bellis, Riccardo Maria Bianchi, Sebastien Binet, Ciril Bohak, Benjamin Couturier, Hadrien Grasland, Oliver Gutsche, Sergey Linev, Alex Martyniuk, Thomas McCauley, Edward Moyse, Alja Mrak Tadel, Mark Neubauer, Jeremi Niedziela, Leo Piilonen, Jim Pivarski, Martin Ritter, Tai Sakuma, Matevz Tadel, and Ben Waugh. 2018. HEP Software Foundation Community White Paper Working Group Visualization. arXiv preprint 1811.10309.

https://arxiv.org/abs/1811.10309

[5] Hrvoje Benko, Edward W. Ishak, and Steven Feiner. 2004. Collaborative mixed reality visualization of an archaeological excavation. In Proc. ISMAR. IEEE Computer Society, Los Alamitos, 132-140. DOI : https://doi .org/10.1109/ISMAR. 2004.23

[6] Lonni Besançon, Paul Issartel, Mehdi Ammi, and Tobias Isenberg. 2017. Hybrid tactile/tangible interaction for 3D data exploration. IEEE Transactions on Visualization and Computer Graphics 23, 1 (Jan. 2017), 881-890. DOI: https://doi.org/10.1109/TVCG. 2016.2599217

[7] Lonni Besançon, Paul Issartel, Mehdi Ammi, and Tobias Isenberg. 2017. Mouse, tactile, and tangible input for 3D manipulation. In Proc. CHI. ACM, New York, 4727-4740. DOI :

https://doi.org/10.1145/3025453.3025863

[8] Lonni Besançon, Amir Semmo, David J. Biau, Bruno Frachet, Virginie Pineau, El Hadi Sariali, Rabah Taouachi, Tobias Isenberg, and Pierre Dragicevic. 2018. Reducing affective responses to surgical images through color manipulation and stylization. In Proc. Expressive. ACM, New York, Article 4, 13 pages. DOI:

https://doi .org/10.1145/3229147.3229158

[9] Lonni Besançon, Mickael Sereno, Mehdi Ammi, Lingyun Yu, and Tobias Isenberg. 2019. Hybrid touch/tangible spatial 3D data selection. Computer
Graphics Forum 38, 3 (June 2019), 553-567. DOI : https://doi.org/10.1111/cgf.13710

[10] Richard Brath. 2014. 3D InfoVis is here to stay: Deal with it. In Proc. 3DVis. IEEE Computer Society, Los Alamitos, 25-31. DOI : https://doi.org/10.1109/3DVis.2014.7160096

[11] Frederik Brudy, Christian Holz, Roman Rädle, Chi-Jui Wu, Steven Houben, Clemens Nylandsted Klokmose, and Nicolai Marquardt. 2019. Cross-device taxonomy: Survey, opportunities and challenges of interactions spanning across multiple devices. In Proc. CHI. ACM, New York, Article 562, 28 pages. DOI: https://doi.org/10.1145/3290605.3300792

[12] Sheelagh Carpendale. 2008. Evaluating information visualizations. In Information Visualization: Human-Centered Issues and Perspectives. LNCS, Vol. 4950. Springer, Berlin/Heidelberg, 19-45. DOI : https://doi.org/10.1007/978-3-540-70956-5_2

[13] Citi. 2016. Citi HoloLens holographic workstation. Online video: https : //www . youtube . com/watch?v=ONogl tmewmQ. (2016). Accessed: August 10, 2019.

[14] Carolina Cruz-Neira, Daniel J. Sandin, Thomas A. DeFanti, Robert V. Kenyon, and John C. Hart. 1992. The CAVE: Audio visual experience automatic virtual environment. Communications of the ACM 35, 6 (June 1992), 64-72. DOI : https://doi.org/10.1145/129888.129892

[15] Gerwin de Haan, Michal Koutek, and Frits H. Post. 2002. Towards intuitive exploration tools for data visualization in VR. In Proc. VRST. ACM, New York, 105-112. DOI : https://doi .org/10.1145/585740.585758

[16] Zach Duer, Leo Piilonen, and George Glasson. 2018. Belle2VR: A virtual-reality visualization of subatomic particle physics in the Belle II experiment. IEEE Computer Graphics and Applications 38 (May/June 2018), 33-43. DOI :

https://doi.org/10.1109/MCG.2018.032421652

[17] Alessandro Febretti, Arthur Nishimoto, Terrance Thigpen, Jonas Talandis, Lance Long, JD Pirtle, Tom Peterka, Alan Verlo, Maxine Brown, Dana Plepys, and others. 2013. CAVE2: A hybrid reality environment for immersive simulation and information analysis. In The Engineering Reality of Virtual Reality (SPIE Proceedings Series), Vol. 8649. SPIE, Bellingham, Washington, Article 864903,12 pages. DOI: https://doi.org/10.1117/12.2005484

[18] Jorge A. Wagner Filho, Carla M. D. S. Freitas, and Luciana Nedel. 2019. Comfortable immersive analytics with the virtualdesk metaphor. IEEE Computer Graphics and Applications 39, 3 (May 2019), 41-53. DOI : https://doi.org/10.1109/MCG.2019.2898856

[19] Andrew Forsberg, Jian Chen, and David Laidlaw. 2009. Comparing 3D vector field visualization methods: A 
user study. IEEE Transactions on Visualization and Computer Graphics 15, 6 (Nov. 2009), 1219-1226. DOI : https://doi.org/10.1109/TVCG.2009.126

[20] Bernd Fröhlich, Stephen Barrass, Björn Zehner, John Plate, and Martin Göbel. 1999. Exploring geo-scientific data in virtual environments. In Proc. Visualization. IEEE Computer Society, Los Alamitos, 169-173. DOI : https://doi.org/10.1109/VISUAL. 1999.809884

[21] Chi-Wing Fu, Wooi-Boon Goh, and Junxiang Allen Ng. 2010. Multi-touch techniques for exploring large-scale 3D astrophysical simulations. In Proc. CHI. ACM, New York, 2213-2222. DOI : https://doi .org/10.1145/1753326.1753661

[22] Jens Grubert, Lukas Witzani, Eyal Ofek, Michel Pahud, Matthias Kranz, and Per Ola Kristensson. 2018. Text Entry in Immersive Head-Mounted Display-based Virtual Reality using Standard Keyboards. arXiv preprint 1802.00626. http://arxiv.org/abs/1802.00626

[23] Juan David Hincapié-Ramos, Xiang Guo, Paymahn Moghadasian, and Pourang Irani. 2014. Consumed endurance: A metric to quantify arm fatigue of mid-air interactions. In Proc. CHI. ACM, New York, 1063-1072. DOI: https://doi .org/10.1145/2556288.2557130

[24] Microsoft HoloLens. 2015. Microsoft hololens: Partner demo with Maya by Autodesk. Online video: https://www. youtube . com/watch?v=q0K3nOGf8mA. (2015). Accessed: August 10, 2019.

[25] Christophe Hurter, Nathalie Henry Riche, Steven M. Drucker, Maxime Cordeil, Richard Alligier, and Romain Vuillemot. 2019. FiberClay: Sculpting three dimensional trajectories to reveal structural insights. IEEE Transactions on Visualization and Computer Graphics 25, 1 (Jan. 2019), 704-714. DOI : https://doi.org/10.1109/TVCG.2018.2865191

[26] Daniel F. Keefe. 2008. Free-form VR interactions in scientific visualization. In Proc. IEEE Visualization Workshop on Scientific Workflow with Immersive Interfaces for Visualization. http://ivlab.cs.umn. edu/ generated/pub-Keefe-2008-FreeFormSciVis.php

[27] Thomas Kittelmann, Vakhtang Tsulaia, Joseph Boudreau, and Edward Moyse. 2010. The Virtual Point 1 event display for the ATLAS experiment. Journal of Physics: Conference Series 219, part 3, Article 032012 (May 2010), 10 pages. DOI : https://doi.org/10.1088/1742-6596/219/3/032012

[28] Tijmen Klein, Florimond Guéniat, Luc Pastur, Frédéric Vernier, and Tobias Isenberg. 2012. A design study of direct-touch interaction for exploratory 3D scientific visualization. Computer Graphics Forum 31, 3 (June 2012), 1225-1234. DOI :

https://doi.org/10.1111/j.1467-8659.2012.03115.x

[29] Wolfgang Krueger and Bernd Froehlich. 1994. The responsive workbench. IEEE Computer Graphics and Applications 14, 3 (May 1994), 12-15. DOI: https://doi.org/10.1109/38.279036
[30] David López, Lora Oehlberg, Candemir Doger, and Tobias Isenberg. 2016. Towards an understanding of mobile touch navigation in a stereoscopic viewing environment for 3D data exploration. IEEE Transactions on Visualization and Computer Graphics 22, 5 (May 2016), 1616-1629. DOI : https://doi.org/10.1109/TVCG.2015.2440233

[31] Claes Lundström, Thomas Rydell, Camilla Forsell, Anders Persson, and Anders Ynnerman. 2011. Multi-touch table system for medical visualization: Application to orthopedic surgery planning. IEEE Transactions on Visualization and Computer Graphics 17, 12 (Dec. 2011), 1775-1784. DOI : https://doi.org/10.1109/TVCG.2011.224

[32] Tahir Mahmood, Erik Butler, Nicholas Davis, Jian Huang, and Aidong Lu. 2018. Building multiple coordinated spaces for effective immersive analytics through distributed cognition. In Proc. BDVA. IEEE Computer Society, Los Alamitos, 119-129. DOI: https://doi .org/10.1109/BDVA. 2018.8533893

[33] Veera Bhadra Harish Mandalika, Alexander I. Chernoglazov, Mark Billinghurst, Christoph Bartneck, Michael A. Hurrell, Niels de Ruiter, Anthony P. H. Butler, and Philip H. Butler. 2018. A hybrid 2D/3D user interface for radiological diagnosis. Journal of Digital Imaging 31, 1 (Feb. 2018), 56-73. DOI: https://doi.org/10.1007/s10278-017-0002-6

[34] G. Elisabeta Marai, Angus G. Forbes, and Andrew Johnson. 2016. Interdisciplinary immersive analytics at the electronic visualization laboratory: Lessons learned and upcoming challenges. In Proc. IA. IEEE Computer Society, Los Alamitos, 54-59. DOI:

https://doi .org/10.1109/IMMERSIVE. 2016.7932384

[35] Stefan Marks, Javier E. Estevez, and Andy M. Connor. 2014. Towards the Holodeck: Fully immersive virtual reality visualisation of scientific and engineering data. In Proc. IVCNZ. ACM, New York, 42-47. DOI: https://doi.org/10.1145/2683405.2683424

[36] Kim Marriott, Falk Schreiber, Tim Dwyer, Karsten Klein, Nathalie Henry Riche, Takayuki Itoh, Wolfgang Stuerzlinger, and Bruce H. Thomas (Eds.). 2018. Immersive Analytics. Springer, Berlin/Heidelberg. DOI : https://doi .org/10.1007/978-3-030-01388-2

[37] John P. McIntire, Paul R. Havig, and Eric E. Geiselman. 2014. Stereoscopic 3D displays and human performance: A comprehensive review. Displays 35, 1 (Jan. 2014), 18-26. DOI : https://doi.org/10.1016/j.displa.2013.10.004

[38] Microsoft. 2019. Microsoft HoloLens: A new way to see your world. Web site: https://www.microsoft. com/en-us/hololens/hardware. (2019). Accessed: Jan. 2019.

[39] Alexandre Millette and Michael J. McGuffin. 2016. DualCAD: Integrating augmented reality with a desktop gui and smartphone interaction. In Proc. 
ISMAR-Adjunct. IEEE, Piscataway, NJ, USA, 21-26. DOI : https://doi .org/10.1109/ISMAR-Adjunct .2016.0030

[40] Ken Nagao, Yucong Ye, Chuan Wang, Issei Fujishiro, and Kwan-Liu Ma. 2016. Enabling interactive scientific data visualization and analysis with see-through HMDs and a large tiled display. In Proc. IA. IEEE Computer Society, Los Alamitos, 1-6. DOI:

https://doi.org/10.1109/IMMERSIVE. 2016.7932374

[41] Jeremi Niedziela and Barthélémy von Haller. 2017. Event visualisation in ALICE - Current status and strategy for Run 3. Journal of Physics: Conference Series 898, 7, Article 072008 (2017), 8 pages. DOI : https://doi .org/10.1088/1742-6596/898/7/072008

[42] Oculus. 2019. Oculus Rift: VR headset for VR ready PCs. Web site: https://www. oculus. com/rift/. (2019). Accessed: Mar. 2019.

[43] Prabhat, Andrew Forsberg, Michael Katzourin, Kristi Wharton, and Mel Slater. 2008. A comparative study of desktop, fishtank, and Cave systems for the exploration of volume rendered confocal data sets. IEEE Transactions on Visualization and Computer Graphics 14, 3 (May 2008), 551-563. DOI : https://doi.org/10.1109/TVCG.2007.70433

[44] Mickael Sereno, Lonni Besançon, and Tobias Isenberg. 2019. Supporting volumetric data visualization and analysis by combining augmented reality visuals with multi-touch input. In Posters EuroVis. 21-23. DOI: https://doi.org/10.2312/eurp. 20191136

[45] Marcos Serrano, Barrett Ens, Xing-Dong Yang, and Pourang Irani. 2015. Gluey: Developing a head-worn display interface to unify the interaction experience in distributed display environments. In Proc. MobileHCI. ACM, New York, 161-171. DOI :

https://doi.org/10.1145/2785830.2785838

[46] Takashi Shibata. 2002. Head mounted display. Displays 23, 1 (April 2002), 57-64. DOI : https://doi .org/10.1016/S0141-9382(02)00010-0

[47] Nicole Sultanum, Sowmya Somanath, Ehud Sharlin, and Mario Costa Sousa. 2011. "Point it, split it, peel it, view it": Techniques for interactive reservoir visualization on tabletops. In Proc. ITS. ACM, New York, 192-201. DOI : https://doi.org/10.1145/2076354.2076390

[48] Erik Sundén, Ingemar Lundgren, and Anders Ynnerman. 2017. Hybrid virtual reality touch table - an immersive collaborative platform for public explanatory use of cultural objects and sites. In Proc. Eurographics Workshop on Graphics and Cultural Heritage. Eurographics Association, Goslar, Germany, 109-113. DOI:https://doi.org/10.2312/gch. 20171300

[49] Ivan E. Sutherland. 1968. A head-mounted three dimensional display. In Proceedings of the Fall Joint Computer Conference, Part I. ACM, New York, 757-764. DOI :

https://doi.org/10.1145/1476589.1476686
[50] Zsolt Szalavári, Dieter Schmalsteig, Anton Fuhrmann, and Michael Gervautz. 1998. "Studierstube": An environment for collaboration in augmented reality. Virtual Reality 3, 1 (March 1998), 37-48. DOI : https://doi .org/10.1007/BF01409796

[51] Rensu P. Theart, Ben Loos, and Thomas R. Niesler. 2017. Virtual reality assisted microscopy data visualization and colocalization analysis. $B M C$ Bioinformatics 18, 2, Article 64 (2017), 16 pages. DOI : https://doi.org/10.1186/s12859-016-1446-2

[52] VIVE. 2019. VIVE: Discover virtual reality beyond imagination. Web site: https://www.vive. com. (2019). Accessed: Mar. 2019.

[53] Ilija Vukotic, Edward Moyse, and Riccardo Maria Bianchi. 2015. ATLASrift - A Virtual Reality application. arXiv preprint 1511.00047. https://arxiv.org/abs/1511.00047

[54] Xiyao Wang, Lonni Besançon, Florimond Guéniat, Mickael Sereno, Mehdi Ammi, and Tobias Isenberg. 2019. A vision of bringing immersive visualization to scientific workflows. In Proc. CHI Workshop on Immersive Analytics. https://hal . archives-ouvertes. fr/hal-02053969

[55] Colin Ware and Glenn Franck. 1996. Evaluating stereo and motion cues for visualizing information nets in three dimensions. ACM Transactions on Graphics 15, 2 (April 1996), 121-140. DOI :

https://doi.org/10.1145/234972.234975

[56] Usher Will, Klacansky Pavol, Federer Frederick, Bremer Peer-Timo, Knoll Aaron, Yarch Jeff, Angelucci Alessandra, and Pascucci Valerio. 2018. A virtual reality visualization tool for neuron tracing. IEEE Transactions on Visualization and Computer Graphics 24, 1 (Jan 2018), 994-1003. DOI :

https://doi.org/10.1109/TVCG.2017.2744079

[57] Graham Wills. 2008. Linked data views. In Handbook of Data Visualization, Chun-houh Chen, Wolfgang Härdle, and Antony Unwin (Eds.). Springer, Berlin/Heidelberg, Chapter II.9, 217-241. DOI : https://doi.org/10.1007/978-3-540-33037-0_10

[58] Bob G. Witmer and Michael J. Singer. 1998. Measuring presence in virtual environments: A presence questionnaire. Presence 7, 3 (June 1998), 225-240. DOI: https://doi.org/10.1162/105474698565686

[59] Lingyun Yu, Konstantinos Efstathiou, Petra Isenberg, and Tobias Isenberg. 2016. CAST: Effective and efficient user interaction for context-aware selection in 3D particle clouds. IEEE Transactions on Visualization and Computer Graphics 22, 1 (Jan. 2016), 886-895. DOI : https://doi .org/10.1109/TVCG. 2015.2467202 